\title{
A NEW MODEL OF MARS ROTATION FROM PATHFINDER DATA
}

\author{
SEBASTIEN BOUQUILLON \& JEAN SOUCHAY \\ Observatoire de Paris, France, E-mail:seb@danof.obspm.fr
}

\begin{abstract}
Following efforts to construct an accurate modelisation of Mars rotation starting from canonical equations in an Hamiltonian theoretical frame (Bouquillon and Souchay, 1996), we use recent results from radio tracking data of the Mars Pathfinder mission (Folkner et al., 1997) to modelize in the best way the motion of precession and nutation of the planet. A complete set of coefficients related to these two motions is presented, including the main effect due to the Sun and also those due to the two satellites Phobos and Deimos as well as to the planets. Morever, Oppolzer terms are calculated and included.
\end{abstract}

\section{The Materialization of the Motion of Rotation}

The canonical variables chosen here in order to solve the equations of motion of Mars by the way of the Hamiltonian equations are the Andoyer variables equivalent to those used in the case of the study of the motion of rotation of the Earth (Kinoshita, 1977). The basic plane $\left(P_{M}^{t}\right)$ is the mean orbit of Mars for the date $t$, which is slightly moving with respect to an inertial plane, which is the mean orbit of Mars $\left(P_{M}^{0}\right)$ at the epoch $\mathrm{J} 2000.0$. The basic point used in order to measure the motion of the precession and nutation in longitude of Mars is the point called the "non-rotating origin" $D_{t}$ along $\left(P_{M}^{t}\right)$ which is described in detail by Guinot(1979) and Capitaine et al. (1986).We choose the non-rotating origin $D_{0}$ at $\mathbf{J} 2000.0$ in coincidence with the equinox $\Gamma_{0}$ of Mars at this epoch. For more details on our materialization of the motion of rotation of Mars, see (Bouquillon and Souchay, 1996).

\section{Pathfinder Data Used in this Study}

With only the two years of Viking data, the observational determination of the precession constant of Mars cannot be done very accurately, as it was shown by Pitjeva(1995), who found a value of $-750 " \pm 36 " / c y$. Nevertheless the recent results from the Mars Pathfinder mission lead to a much more accurate determination of the precession, that is to say $-757.6 \pm 3.5^{\prime \prime} / c y$ (Folkner et al.,1997). So, this more accurate determination reduces the uncertainly of the dynamical ellipticity: $H_{d}=$ $0.005363 \pm 25$ which has important effects for the accuracy of the determination of nutation coefficients.

\section{Results}

The series of nutations are calculated with the help of VSOP87, (see Bretagnon and Francou, 1988) for the motion of Mars and the other planets, and with ESAPHO and ESADE (see Chapront-touzé, 1990) for the motion of Phobos and Deimos. These series are truncated at 0.01 mas. These series are available from the authors ( Bouquillon and Souchay, 1998). 


\section{References}

Bouquillon S., Souchay J.:1996, Proceeding of I.A.U colloquium 165.

Bouquillon S., Souchay J.:1998, Astron. Astrophys., in preparation

Bretagnon, P., Francou, G.,1988, Astron. Astrophys., 202,309

Capitaine, N., Guinot, B., and Souchay, J.: 1986, Celest. Mech., 39, 283.

Chapront-Touzé, M.: 1990, Astron. Astrophys., 240, 159.

Folkner, W.M., Yoder, C.F., Yuan,D.N., Standish, E.M., Preston, R.A., 1997, Science, 278, 1749

Guinot, B.: 1979, "Basic problems in the kinematics of the rotation of the Earth", in: Time and the Earth's Rotation 7-8 (D.D. McCarthy, J.D. Pilkington, eds), Reidel, Dordrecht .

Kinoshita, H.: 1977, Celest. Mech., 26, 296.

Pitjeva E.V.: 1995, IA.U. symposium 172, (S.Ferraz-Mello, B.Morando and J.-E.Arlot, eds), 45. 